\title{
Cycle control with an extended-regimen oral contraceptive combining levonorgestrel and ethinyl estradiol that includes continuous low- dose ethinyl estradiol instead of the traditional hormone-free interval
}

This article was published in the following Dove Press journal:

International Journal of Women's Health

5 October 2017

Number of times this article has been viewed

Rossella E Nappi'

Paloma Lobo Abascal ${ }^{2}$

Jennifer Hsieh ${ }^{3}$

Marie-Christine Micheletti ${ }^{4}$

'Research Center for Reproductive Medicine, Gynecological

Endocrinology and Menopause, IRCCS San Matteo Foundation, Department of Clinical, Surgical, Diagnostic and Pediatric Sciences, University of Pavia, Pavia, Italy; ${ }^{2}$ Hospital Universitario Infanta Sofía, San Sebastián de los Reyes, Madrid, Spain; ${ }^{3}$ Teva Branded Pharmaceutical Products R\&D, Inc., Malvern, PA, USA; ${ }^{4}$ Teva Europe Women's Health Medical Affairs, Amsterdam, the Netherlands
Correspondence: Rossella E Nappi Research Center for Reproductive Medicine, Gynecological Endocrinology and Menopause, Department of Obstetrics and Gynecology, IRCCS San Matteo Foundation, University of Pavia, Piazzale Golgi 2, 27100 Pavia, Italy Tel +390382501561 Fax +390382536176 Email renappi@tin.it
Purpose: To evaluate scheduled and unscheduled bleeding and spotting over 1 year of treatment with 91-day extended-regimen combined oral contraception (COC) providing continuous lowdose ethinyl estradiol (EE) in place of the traditional 7-day hormone-free interval (HFI).

Patients and methods: This post hoc analysis of a multicenter, open-label, 1-year, Phase 3 study of extended-regimen COC with $30 \mu \mathrm{g} \mathrm{EE} / 150 \mu \mathrm{g}$ levonorgestrel (LNG) for 84 days and EE $10 \mu \mathrm{g}$ for 7 days included 799 sexually active, adult women who completed at least one 91-day cycle of therapy. Subjects recorded bleeding and spotting episodes daily using electronic diaries. Logistic regression analyses are reported as ORs with 95\% CIs.

Results: There was a $10 \%$ increase $(\mathrm{OR}=1.102$; $95 \% \mathrm{CI}$ : $1.006-1.206)$ in the likelihood of reporting no scheduled bleeding for each additional 91-day cycle completed. From the third 91-day cycle, more than one fifth of women reported no scheduled bleeding (third cycle $=23 \%$ [121/533]; fourth cycle $=22 \%$ [97/446]). Among women who reported no scheduled bleeding at Cycle 1 (136/758 [18\%]), $\geq 45 \%$ showed sustained lack of scheduled bleeding in later cycles. There were increases of 53\% (OR $=1.531$; 95\% CI: $1.393-1.683)$ and $31 \%(\mathrm{OR}=1.307$; 95\% CI: $1.205-1.418$ ) in the likelihood of reporting 0 to $\leq 6$ days vs $>6$ days of unscheduled bleeding and spotting, respectively, for each additional 91-day cycle. By Cycle 2, more than $80 \%$ of women reported no unscheduled bleeding or $\leq 6$ days of unscheduled bleeding during each 91-day cycle.

Conclusion: Improved cycle control with decreased bleeding over time was shown during extended-regimen COC with $30 \mu \mathrm{g}$ EE/150 $\mu \mathrm{g}$ LNG for 84 days and continuous low-dose EE instead of the traditional 7-day HFI. Women considering this regimen should be informed that those who complete at least one 91-day COC cycle will likely experience less bleeding/ spotting in future cycles.

Keywords: combined oral contraception, menstrual cycle, 91-day cycle, extended-regimen $\mathrm{COC}$

\section{Introduction}

Combined oral contraception (COC) continues to be the method most frequently selected by women using hormonal contraception in the United States and Europe. ${ }^{1,2}$ Monthly cyclic regimens, including 21/7 or 24/4 regimens, remain the most common; however, use of extended-regimen $\mathrm{COC}$ is increasing. ${ }^{3} \mathrm{COC}$ extended regimens include 
greater than 28 days of active pills, reduce the number of scheduled bleeding episodes, and may maintain, modify, or eliminate the usual 7-day hormone-free interval (HFI) that occurs within the traditional 28 -day regimen. ${ }^{4}$ The utility of extended-regimen COC for women may include improvement in medical conditions, such as premenstrual syndrome (PMS), dysmenorrhea, menstrually-related headaches, endometriosis, iron deficiency, and anemia, as well as reduced inconvenience and interference with daily activities, work/ school attendance, personal social events, and sports; overall improved quality of life (QOL); and economic benefits related to reduced expenses for feminine products and reduced use of pain medication., ${ }^{5,6}$ Additionally, menstrual cycle-related symptoms and hormone withdrawal symptoms have shown greater improvement during extended or continuous regimens vs $21 / 7$ or 24/4 regimens. ${ }^{5,6}$ Importantly, benefit from extended-regimen $\mathrm{COC}$ is not limited to women with menstrual cycle-related symptoms or disorders, as regular monthly bleeding has also been reported to have severe negative impact on daily life. ${ }^{7}$

Surveys examining bleeding preferences and attitudes about menstrual suppression show that the majority of women prefer menstrual periods at a frequency of less than once monthly, ${ }^{8-10}$ and instead prefer once every 3 months or no periods at all. ${ }^{8,9,11}$ Key non-medical factors underlying desire for menstrual suppression include convenience and reducing interference with sexuality, social life, work, and sports activities. $^{8-10}$ Widespread acceptance of extended-regimen COC by clinicians has been shown. ${ }^{12}$ Approximately $75 \%$ to almost $100 \%$ of surveyed health care providers (HCPs) report discussion of extended-regimen $\mathrm{COC}$ with patients or prescription of extended-regimen $\mathrm{COC}$ at least once. ${ }^{13-16}$ Common reasons given by HCPs for prescribing extendedregimen $\mathrm{COC}$ include convenience of menstrual suppression and relief from heavy bleeding, menstrual-related headaches, dysmenorrhea/pelvic pain, endometriosis, and PMS. ${ }^{3,14-16}$

The 91-day extended-regimen COC with $30 \mu \mathrm{g}$ ethinyl estradiol (EE) $/ 150 \mu \mathrm{g}$ levonorgestrel (LNG) for 84 days and EE $10 \mu \mathrm{g}$ for 7 days during the typical HFI (Seasonique ${ }^{\circledR}$; Teva Branded Pharmaceutical Products R\&D, Inc, Malvern, PA, USA) was approved by the US Food and Drug Administration in 2006 and was approved by the European Medicines Agency in 2015. ${ }^{17}$ The inclusion of continuous low-dose EE rather than the HFI may help address the suboptimal suppression of ovarian activity that has been shown with low-dose COC that includes the traditional 7-day HFI. ${ }^{18,19}$ Additionally, greater ovarian suppression may result in improved cycle control and reduced risk of unscheduled bleeding. ${ }^{20,21}$
This 91-day extended-regimen COC with continuous lowdose EE has only four scheduled bleeds per year that, on average, last approximately 3 days and are light ( 1 of 3 days is limited to spotting). ${ }^{22}$ With extended-regimen COC, some women may experience even less scheduled bleeding and some women may experience unscheduled bleeding. ${ }^{21}$

Because cycle control plays an important role in COC satisfaction and continuation, ${ }^{23,24}$ closer evaluation of cycle control over time is needed in order to provide optimal contraceptive counseling for women who are considering extended-regimen COC. This post hoc analysis of an earlier Phase 3 study evaluated changes in scheduled and unscheduled bleeding and spotting over 12 months in women receiving 84/7 LNG/EE extended-regimen COC with 7 days of low-dose EE in place of the traditional HFI. ${ }^{22}$ Whereas the Phase 3 study reported mean and median days of bleeding/spotting by cycle, the current analysis provides a more detailed examination of: a) the likelihood of change in scheduled and unscheduled bleeding/spotting across the four 91-day cycles, b) whether the absence of scheduled bleeding in some women during the first cycle is sustained across cycles, c) the relationship between absence of scheduled bleeding and the experience of unscheduled bleeding/ spotting, and d) the association of participant characteristics (COC use history, smoking, body mass index [BMI], and age) with unscheduled bleeding/spotting.

\section{Patients and methods}

\section{Study design}

This study is a post hoc analysis of the multicenter, openlabel, 1-year, Phase 3 study which examined the efficacy and safety of 91-day extended-regimen COC that includes $30 \mu \mathrm{g}$ EE $/ 150 \mu \mathrm{g}$ LNG for 84 days and EE $10 \mu \mathrm{g}$ for 7 days during the traditional HFI. ${ }^{22}$ Our post hoc analysis evaluated scheduled and unscheduled bleeding and spotting in participants over 12 months. The Phase 3 study received ethics committee approval at each clinical site, all participants signed informed consent, and the study was conducted in accordance with the Declaration of Helsinki. The 1-year study included four consecutive cycles of 91-day extendedregimen COC. Participants included women with continuous COC use who switched from another COC regimen (ie, continuous), women who had no prior history of COC use (ie, new starters), and women who had a history of COC use without any COC use in the 6 months prior to study enrollment (ie, prior users). The Phase 3 study included sexually active, adult women aged 18 to 40 years who were seeking COC as their primary birth control method. 
Active smokers were excluded if they were older than 35 years. $^{22}$ This post hoc analysis included 799 women in the Phase 3 study who completed at least one cycle of 91-day extended-regimen COC. To be included in the analysis for each respective 91-day cycle, study subjects were also required to have electronic diary data for bleeding/spotting for the completed cycle.

\section{Assessments}

Women recorded bleeding and spotting episodes daily using electronic diaries with preprogrammed questions. Women could only enter information for a given day between 12:00 am and 11:59 pm of that day. The electronic diary included an alarm set to each participant's scheduled pill time, which also served as a reminder to complete the diary. Bleeding was defined as vaginal blood loss that required sanitary protection. Spotting was defined as vaginal blood loss that did not require sanitary protection. Scheduled bleeding/ spotting occurred on cycle Days 85 to 91 and unscheduled bleeding/spotting occurred on cycle Days 1 to 84. Any scheduled bleeding or spotting that continued into Day 1 or longer in the next cycle was counted as unscheduled bleeding or spotting for that cycle, which may have contributed to an increased count of unscheduled bleeding days vs scheduled bleeding days in each cycle. This approach provides a "worst case" scenario for unscheduled bleeding, which is typically reported as most distressing by women. Cycle 1 extendedregimen COC started on the Sunday following the first day of each women's menstrual cycle. During the first 7 days of Cycle 1, any reported bleeding/spotting days were excluded from the Cycle 1 count of unscheduled bleeding/spotting.

\section{Data analysis}

Logistic regression analyses were conducted to evaluate changes in scheduled and unscheduled bleeding/spotting with each 91-day cycle completed and ORs with 95\% CIs are reported. Among women who reported no scheduled bleeding at Cycle 1, descriptive analyses examined whether no scheduled bleeding was sustained over subsequent cycles, and among women who reported no scheduled spotting at Cycle 1, descriptive analyses examined whether no scheduled spotting was sustained over subsequent cycles. Descriptive analyses examined the number of unscheduled bleeding/spotting days in women who experienced scheduled bleeding at Cycle 4 compared with women who did not experience scheduled bleeding at Cycle 4. Exploratory subgroup analyses using chi-square tests further examined unscheduled bleeding/spotting by COC use history, smoker vs non-smoker, BMI $\left(<25 \mathrm{~kg} / \mathrm{m}^{2}\right.$ vs $\left.\geq 25 \mathrm{~kg} / \mathrm{m}^{2}\right)$, and age ( $<25$ years, 25 to 35 years, $>35$ years).

\section{Results}

The Phase 3 study included 799 women who completed at least one extended-regimen COC cycle (intent-to-treat cohort) of $30 \mu \mathrm{g} \mathrm{EE} / 150 \mu \mathrm{g}$ LNG for 84 days and EE $10 \mu \mathrm{g}$ for 7 days. The mean age of the cohort was $27.5 \pm 5.71$ years (range 18.0-40.2 years), 80\% (641/799) of the women were Caucasian, and 18\% (147/799) were smokers. The mean BMI was $25.9 \pm 6.07 \mathrm{~kg} / \mathrm{m}^{2}$ (range $17.1-56.5 \mathrm{~kg} / \mathrm{m}^{2}$ ); $53 \%(427 / 798)$ of the women had a BMI $<25 \mathrm{~kg} / \mathrm{m}^{2}$ indicating normal weight, 27\% (216/798) had a BMI $\geq 25$ but $<30 \mathrm{~kg} / \mathrm{m}^{2}$ indicating overweight, and 19\% (155/798) had a BMI $\geq 30 \mathrm{~kg} / \mathrm{m}^{2}$ indicating obesity. The majority of women were continuous COC users (69\% [548/799]), followed by prior COC users (22\% [175/799]), and 10\% (76/799) were COC new starters. Of these 799 women, the current post hoc analysis included only those with bleeding/ spotting electronic diary data for the cycle analyzed, including 758 women in Cycle 1, 625 women in Cycle 2, 533 women in Cycle 3, and 446 women in Cycle 4. Examination of study discontinuation showed 10\% (80/799) of women discontinued due to an adverse event, with 4\% (32/799) due to bleeding/spotting-related AEs.

\section{Scheduled bleeding or spotting}

There was a $10 \%$ increase in the likelihood of reporting no scheduled bleeding during cycle days 85 to 91 for each additional 91-day cycle completed (OR $=1.102$ [95\% CI: 1.006-1.206]; Figure 1A). By Cycle 3 (23\% [121/533] of women) and Cycle 4 (22\% [97/446] of women) more than one in five women reported no scheduled bleeding. There was a $9 \%$ increase in the likelihood of reporting no scheduled spotting for each additional 91-day cycle completed $(\mathrm{OR}=1.085$ [95\% CI: 1.005-1.170]), with 65\% (289/446) of women reporting no scheduled spotting by Cycle 4 (Figure 1B). Among women who reported no scheduled bleeding at Cycle 1 (136/758 [18\%]), $\geq 45 \%$ showed sustained lack of scheduled bleeding in the subsequent cycles (Cycle 2=45\% [45/101 women who also completed Cycle 2], Cycle 3=50\% [41/82 women who also completed Cycle 3], Cycle 4=46\% [36/78 women who also completed Cycle 4]). Among women who reported no scheduled spotting at Cycle 1 (458/758 [60\%]), $\geq 63 \%$ showed sustained lack of scheduled spotting in each of the subsequent cycles (Cycle 2=69\% [256/373 women]; Cycle 3=72\% [231/319 women]; Cycle 4=63\% [189/299 women]). 

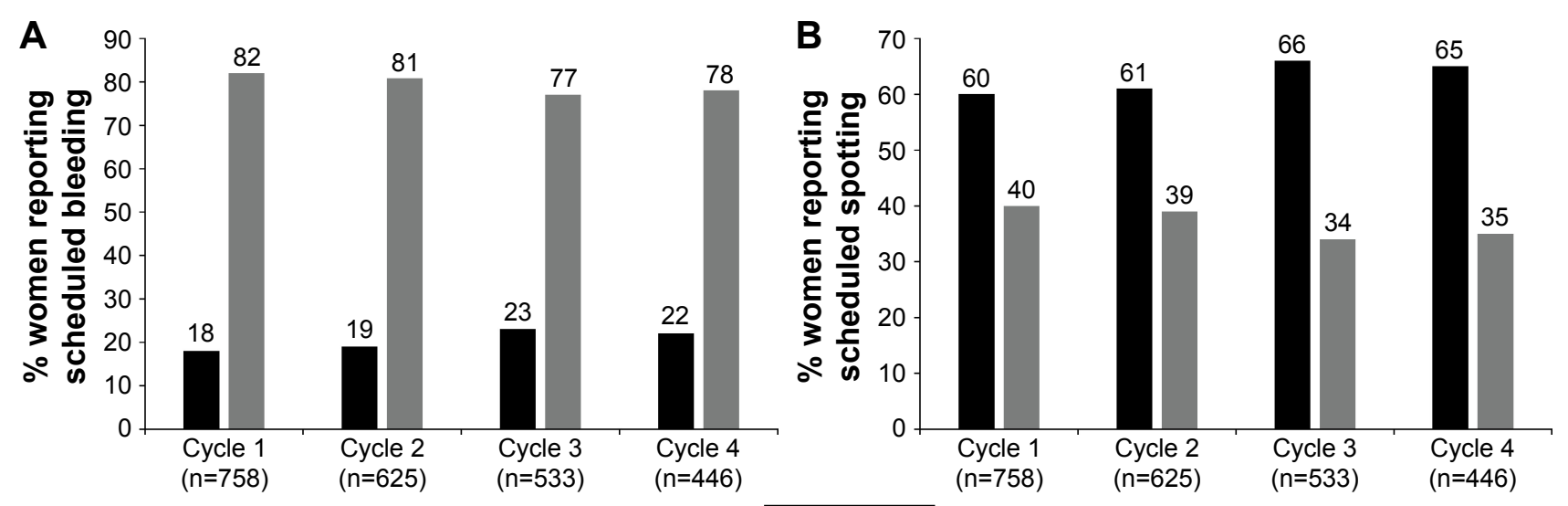

- No Yes

Figure I Percentage of women reporting the occurrence of scheduled bleeding $(\mathbf{A})$ or spotting $(\mathbf{B})$ during each extended-regimen 91 -day cycle.

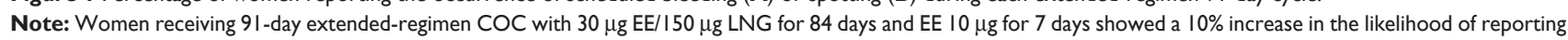
no scheduled bleeding (OR =I.I02 [95\% Cl: I.006-I.206]) and a 9\% increase in the likelihood of reporting no scheduled spotting (OR =I.085 [95\% Cl: I.005-I.I70]) for each additional 91 -day cycle completed.

Abbreviations: COC, combined oral contraception; EE, ethinyl estradiol; LNG, levonorgestrel.

\section{Unscheduled bleeding or spotting}

There was a $53 \%$ increase in the likelihood of reporting 0 to $\leq 6$ days vs $>6$ days of unscheduled bleeding during cycle days 1 to 84 for each additional 91 -day cycle $(\mathrm{OR}=1.531$ [95\% CI: 1.393-1.683]; Figure 2). The percentage of women reporting $>6$ days of unscheduled bleeding during the 91-day cycle decreased to $15 \%$ by both the third ( $81 / 533$ women) and fourth $(69 / 446$ women) cycles. There was a $31 \%$ increase in the odds of reporting 0 to $\leq 6$ days vs $>6$ days of unscheduled spotting for each additional 91-day cycle ( $\mathrm{OR}=1.307$ [95\% CI: 1.205-1.418]; Figure 3). The percentage of women

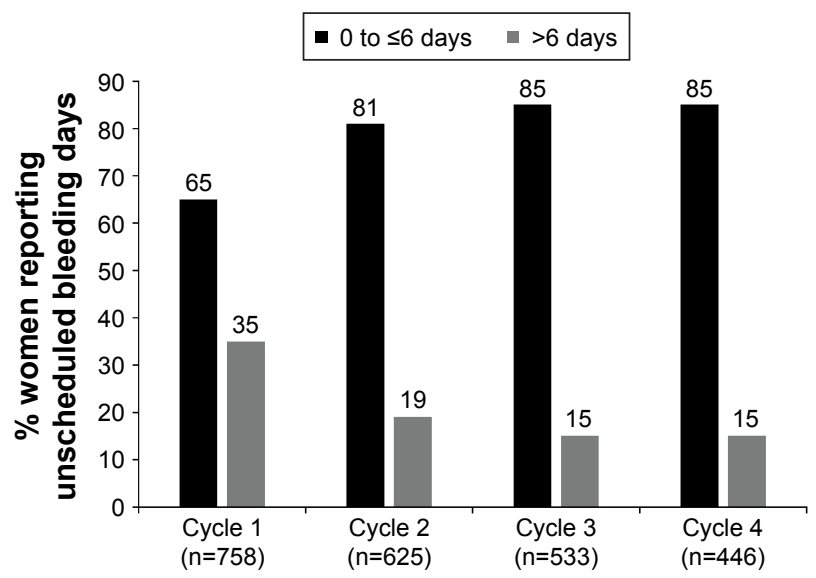

Figure 2 Percentage of women reporting 0 to $\leq 6$ days or $>6$ days of unscheduled bleeding during each extended-regimen 91 -day cycle.

Note: Women receiving $9 \mathrm{I}$-day extended-regimen COC with $30 \mu \mathrm{gE} / \mathrm{I} 50 \mu \mathrm{g}$ LNG for 84 days and EE $10 \mu \mathrm{g}$ for 7 days showed a $53 \%$ increase in the likelihood of reporting 0 to $\leq 6$ days of unscheduled bleeding per 9 I-day cycle (OR $=1.53$ I [95\% Cl: 1.393-1.683]).

Abbreviations: COC, combined oral contraception; EE, ethinyl estradiol; LNG, levonorgestrel. reporting $>6$ days of unscheduled spotting decreased to $24 \%(128 / 533)$ by the third cycle and $25 \%(113 / 446)$ by the fourth 91-day cycle. The percentage of women reporting no unscheduled bleeding increased from 36\% (273/758) in Cycle 1 to $42 \%(222 / 533)$ by Cycle 3 and 39\% (175/446) by Cycle 4 , and the percentage of women reporting no unscheduled bleeding and no unscheduled spotting was $18 \%$ (137/758) in Cycle 1, 18\% (97/533) at Cycle 3, and 19\% (83/446) at Cycle 4.

The relationship between scheduled bleeding/spotting and unscheduled bleeding/spotting was examined during

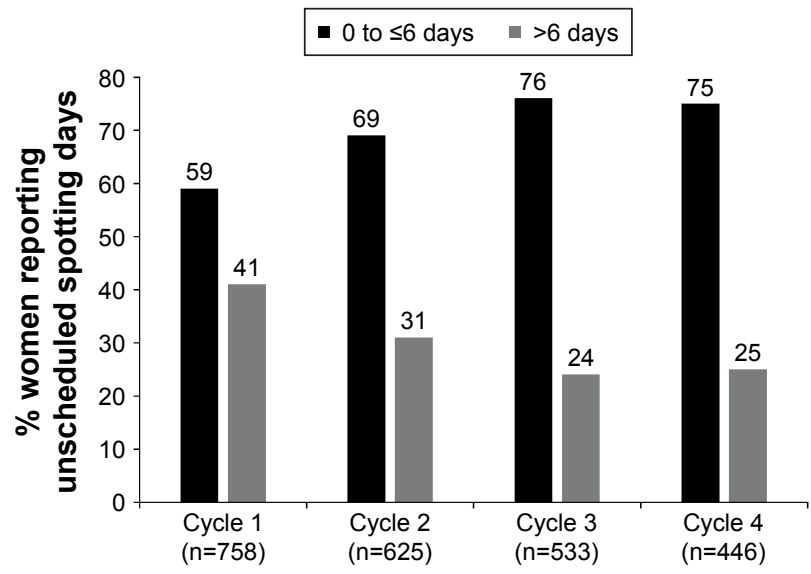

Figure 3 Percentage of women reporting 0 to $\leq 6$ days or $>6$ days of unscheduled spotting during each extended-regimen 91 -day cycle.

Note: Women receiving 9I-day extended-regimen COC with $30 \mu \mathrm{gE} / \mathrm{I} 50 \mu \mathrm{g}$ LNG for 84 days and EE $10 \mu \mathrm{g}$ for 7 days showed a $31 \%$ increase in the odds of reporting 0 to $\leq 6$ days of unscheduled spotting per 9 I-day cycle (OR $=1.307$ [95\% Cl: $1.205-1.418])$.

Abbreviations: COC, combined oral contraception; EE, ethinyl estradiol; LNG, levonorgestrel. 
the fourth 91-day cycle and, important for counseling, showed women who had absence of scheduled bleeding did not have greater unscheduled bleeding. Women who did not experience scheduled bleeding/spotting during Cycle 4 $(n=80)$ reported an average of $4.1 \pm 6.1$ days (Median $=2.0)$ of unscheduled bleeding or spotting. Women who did experience scheduled bleeding/spotting during Cycle $4(n=366)$ reported an average of $8.3 \pm 9.8$ days (Median $=4.0$ ) of unscheduled bleeding or spotting during the 91-day cycle.

Evaluation of unscheduled bleeding/spotting by subgroups (Figure 4) found significantly more women in the continuous COC use group (42\% [226/536]) reported no unscheduled bleeding in the first 91-day cycle compared with women in the COC new starters $(27 \%$ [17/63]) and prior COC user (19\% [30/159]) groups $(P<0.001)$. The COC use groups did not significantly differ in their report of no unscheduled bleeding days in the 91-day Cycles 2 through 4, and by the fourth cycle, $39 \%$ of women who were continuous COC users (129/333), 43\% (13/30) of new starters, and $40 \%(33 / 83)$ of women with prior use of COC reported no unscheduled bleeding. Similarly, significantly more women in the continuous COC use group (28\% [151/536] vs $16 \%$ [10/63] new starters and $16 \%$ [26/159] prior users; $P=0.002$ ) reported no unscheduled spotting in Cycle 1 and there were no significant differences among COC groups in report of unscheduled spotting during Cycles 2-4. By Cycle 4, $32 \%$ (108/333) of continuous COC users, 30\% (9/30) of COC new starters, and 40\% (33/83) of prior COC users reported no unscheduled spotting.

Non-smoking women and women who smoked did not significantly differ in report of unscheduled bleeding across treatment cycles. At Cycle 1, 37\% (232/625) of nonsmoking women reported no unscheduled bleeding and $39 \%$ (148/378) reported no unscheduled bleeding at Cycle 4. Among women who smoked, 31\% (41/133) reported no unscheduled bleeding at Cycle 1 and 40\% (27/68) reported no unscheduled bleeding at Cycle 4 (Figure 4). The nonsmoking and smoking women did not significantly differ in their report of no unscheduled spotting days across treatment cycles.
A

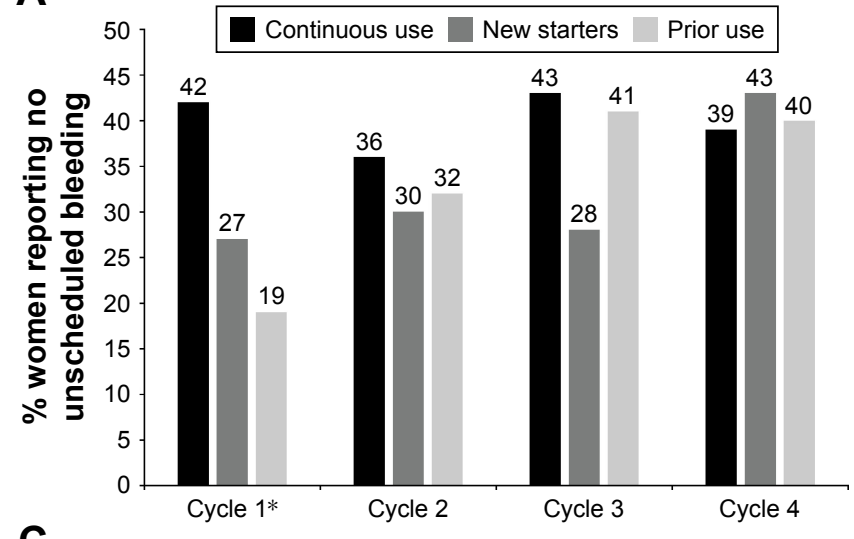

C

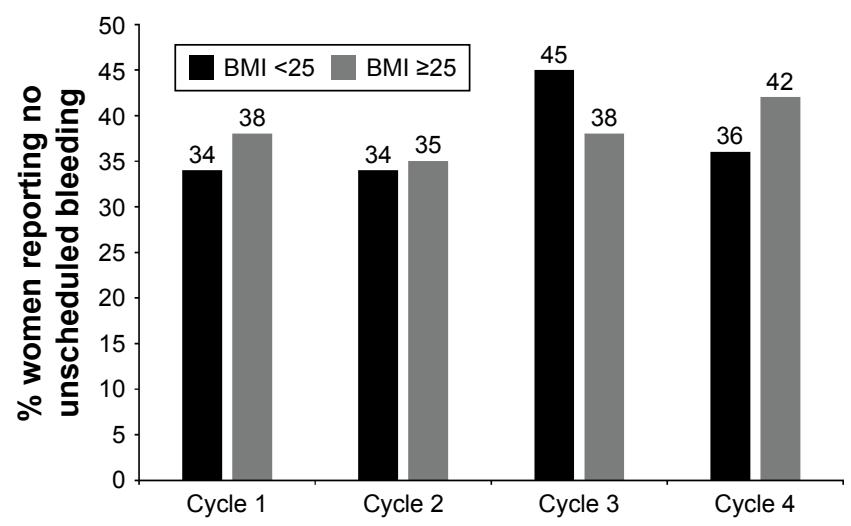

B

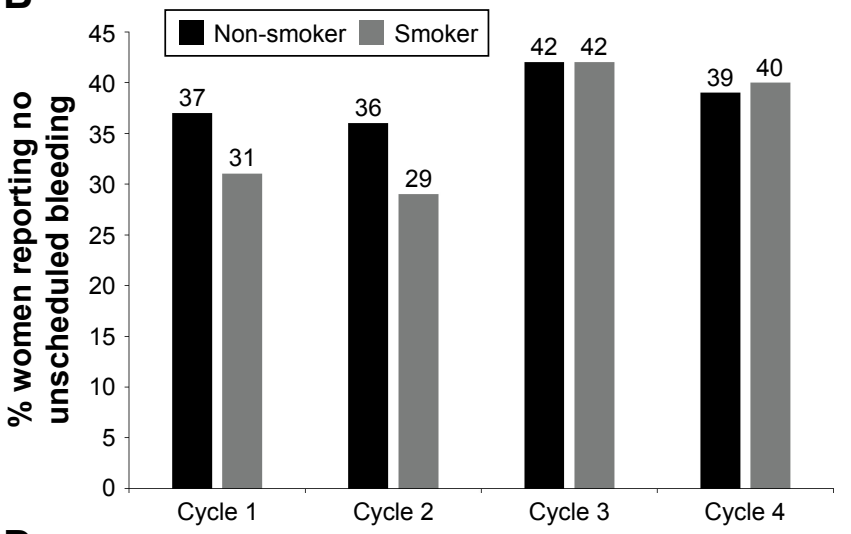

D

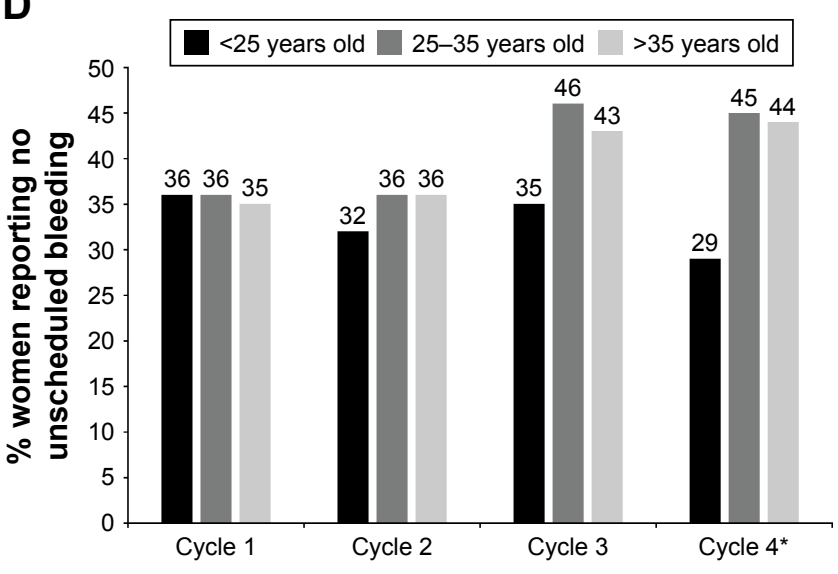

Figure 4 Percentage of women reporting no unscheduled bleeding during each extended-regimen 9I-day cycle by COC use history (A), smoker status (B), BMI (C), and age (D).

Note: *Chi-square test, $P<0.05$.

Abbreviations: COC, combined oral contraception; BMI, body mass index. 
There were no significant differences between BMI groups in report of unscheduled bleeding across the treatment cycles. The percentage of women with BMI $<25 \mathrm{~kg} / \mathrm{m}^{2}$ who reported no unscheduled bleeding was 34\% (138/405) in Cycle 1 and 36\% (83/228) in Cycle 4, and among women with BMI $\geq 25 \mathrm{~kg} / \mathrm{m}^{2}$ was $38 \%$ (135/352) in Cycle 1 and $42 \%$ (92/218) in Cycle 4 (Figure 4). There were no significant differences in unscheduled spotting by BMI groups across cycles.

Examination of unscheduled bleeding by age groups showed similar percentages of women reporting no unscheduled bleeding during Cycles 1-3 (Figure 4). During Cycle 4, a lower percentage of women $<25$ years old reported no unscheduled bleeding $(29 \%, 45 / 154)$ compared with women 25-35 years old or $>35$ years old (45\% [103/230] and $44 \%$ [27/62], respectively; $P=0.007)$. There were no significant differences in unscheduled spotting by age groups across cycles.

\section{Discussion}

In this post hoc analysis of a multicenter, open-label, 1-year, Phase 3 study of 91-day extended-regimen COC that provides $30 \mu \mathrm{g} \mathrm{EE} / 150 \mu \mathrm{g}$ LNG for 84 days and EE $10 \mu \mathrm{g}$ for 7 days during the traditional $\mathrm{HFI},{ }^{22}$ we found an increase in report of no scheduled bleeding/spotting from the first to the fourth 91-day cycle, with more than one in five women reporting no scheduled bleeding during the third and fourth cycles. Among women with no scheduled bleeding at Cycle 1, absence of scheduled bleeding was sustained through subsequent cycles by $\geq 45 \%$ of these women. Among women with no scheduled spotting in Cycle 1, $\geq 63 \%$ showed sustained absence in subsequent cycles. Moreover, women without scheduled bleeding/spotting at Cycle 4 reported, on average, 50\% fewer unscheduled bleeding/spotting days compared with women who did experience scheduled bleeding/spotting. This supports that women experiencing absence of scheduled bleeding do not experience a compensatory increase in unscheduled bleeding.

We also found decreased report of unscheduled bleeding/ spotting over time. By the second cycle, approximately eight in every ten women reported no unscheduled bleeding or $\leq 6$ days of unscheduled bleeding during each 91-day cycle. More than two thirds of women reported no unscheduled spotting or $\leq 6$ days of unscheduled spotting by the second 91-day cycle, which increased to three fourths of women by the fourth cycle. The acceptability of the bleeding pattern of this extended-regimen $\mathrm{COC}$ is further supported by the evidence that only $4 \%$ of women discontinued due to bleeding/spotting-related AEs.
Our findings reinforce the evidence that extendedregimen 91-day cycle COC use with low-dose EE during the traditional HFI, with quarterly scheduled bleeding, is a contraceptive option that helps to meet an important treatment need among the growing number of women who desire fewer scheduled bleeding episodes. ${ }^{7,8}$ Effectiveness of extended-regimen $84 / 7 \mathrm{COC}$ as a contraceptive method in the real world has been shown through significantly lower unintended pregnancy rates compared with $21 / 7$ or $24 / 4$ regimens, using an insurance claims database..$^{25}$ Efficacy has been well demonstrated with the 84/7 LNG/EE regimen with continuous low-dose EE through effective unintended pregnancy prevention, ${ }^{22}$ and significantly decreased follicle stimulating hormone, estradiol, and developing follicles vs 7-day HFI regimens, ${ }^{19}$ as well as effective suppression of ovarian activity and ovulation inhibition. ${ }^{26}$ The safety of this extended-regimen $\mathrm{COC}$ is supported by studies showing no further accumulation of hormones, ${ }^{27}$ no unexpected changes in the endometrium or endometrial hyperplasia, ${ }^{28}$ no effect of cycle length on hemostatic biomarkers, ${ }^{29}$ and similar incidence rates of adverse events over 4 years of continuous use $^{30}$ compared with the conventional 28 -day cycle.

The bleeding patterns reported by women using this extended regimen in our post hoc analysis align well with the preference being expressed by many women for reduced bleeding episodes and increased acceptance of contraceptiveinduced amenorrhea. ${ }^{8,11}$ The replacement of the traditional HFI with continuous low-dose EE in this regimen may further improve hormone withdrawal symptoms experienced by some women ${ }^{18}$ and may improve cycle control, including unscheduled bleeding/spotting. The improvement may happen earlier/sooner following initiation of treatment compared with $84 / 7$ regimens that include the 7 -day HFI, ${ }^{21}$ possibly through greater ovarian suppression associated with removal of the HFI. ${ }^{19}$

Our findings contribute much needed information for contraceptive counseling related to cycle control expectations among women who are interested in initiating this 91-day extended-regimen COC with continuous low-dose EE. Women considering this $\mathrm{COC}$ regimen should be informed that those who complete at least one 91-day cycle would likely experience less bleeding in future cycles and that they should be patient during the first cycle (ie, first 3 months) as they adapt to the regimen. Counseling related to cycle control should address appropriate expectations for scheduled and unscheduled bleeding during the first and subsequent 91-day treatment cycles. ${ }^{12}$ During Cycle 1, our analysis suggests almost one in five women will have no scheduled bleeding 
and six in ten women will have no scheduled spotting, and approximately two thirds of women will have $\leq 6$ days of unscheduled bleeding, including approximately one third of these women with no unscheduled bleeding. Our analysis shows that rather than four scheduled bleeding episodes per year, by the third and fourth cycles, more than one in five women may not experience scheduled bleeding. For those women who do not experience scheduled bleeding in the first cycle, the majority will likely sustain a lack of scheduled bleeding in subsequent cycles. Finally, among women who do not experience scheduled bleeding in the fourth cycle, these women will likely also experience fewer days of unscheduled bleeding/spotting and will not experience any compensatory increase in unscheduled bleeding/spotting.

Related to counseling about unscheduled bleeding/ spotting, our exploratory analyses found women who were new users of $\mathrm{COC}$, or returning to COC use after at least 6 months without COC use were more likely to experience unscheduled bleeding or spotting compared with women who were continuing $\mathrm{COC}$ use during the first treatment cycle, but this difference was not sustained in later treatment cycles. Whereas the percentage of women 25-35 years old or $>35$ years old reporting no unscheduled bleeding increased from the first to the fourth treatment cycle, women younger than 25 years old did not show this same increase. Thus, these groups of women need specific counseling directed at appropriate cycle control expectations. Our analyses did not support a need to tailor counseling specific to BMI or smoking status.

Women can be reassured that for most, the number of days of scheduled and unscheduled bleeding will decrease by the second cycle and may continue to decrease through the first year of treatment. Additionally, the finding that bleeding patterns have been shown to be similar or improved over time for extended regimens vs 28-day regimens ${ }^{5}$ can be shared with patients. Women should be educated that the most common cause of unscheduled bleeding is missed or delayed pills, and the importance of a pill-taking routine that maximizes adherence to the regimen should be emphasized. ${ }^{24}$

In line with "contingency counseling", ${ }^{31}$ the goal of counseling women about the types of bleeding patterns to be expected with extended-regimen COC is to help prepare them for the possibility of no scheduled bleeding, to help them effectively manage unscheduled or unanticipated bleeding and help them understand such a bleeding pattern is likely temporary, and to decrease the likelihood of early discontinuation. It is well documented that some women prefer regularly scheduled bleeding and consider absence of scheduled bleeding highly undesirable, and counseling about possible absence of scheduled bleeding with particular regimens will be especially important for these women. ${ }^{11,32}$ The balance that each woman will need to consider with extended-regimen $\mathrm{COC}$ is the high satisfaction reported with reduced bleeding overall, improvement in menstrual cycle-related symptoms and disorders, and reduction in inconvenience and interference with daily activities vs the individual significance of potential unscheduled bleeding or spotting, particularly in the earliest cycles of treatment. ${ }^{4,5,12,24}$ High satisfaction and improved QOL may overcome the inconvenience of unscheduled bleeding or spotting, as shown among women with menstrual cycle-related symptoms who received extended-regimen $\mathrm{COC}$ and chose to continue the regimen, even with persistent breakthrough bleeding or spotting episodes. ${ }^{33}$

Individualization of contraception counseling is critical, allowing understanding of personal or cultural beliefs that may influence preferences for the timing of bleeding episodes and affect contraceptive method satisfaction and continuation. ${ }^{6,7}$ Counseling should empower patients to discuss individual preferences and weigh personal risks and benefits of contraception options. ${ }^{34}$ Additionally, counseling should include identifying any misconceptions and educating patients that monthly bleeding is not necessary and is not an indication of health when using COC, distinguishing between the menstrual cycle and periodic artificial scheduled bleeding with $\mathrm{COC}$, and addressing any concerns about future fertility. The aim is to help patients understand the acceptability, safety, and potential non-contraceptive benefits of extended-regimen COC. , $, 9,16,34$ HCPs should help patients identify their preferences (eg, preferred bleeding schedule, non-contraceptive benefits) and assist them in their assessment of the acceptability of possible side effects and any concerns a patient has regarding specific side effects. ${ }^{31,35}$

Strengths of this post hoc analysis include the large sample size of women with bleeding data for each of the completed 91-day COC cycles. Additionally, the collection of daily bleeding information using electronic diaries may have improved accuracy in reporting compared with paper diaries. A limitation of the Phase 3 study on which this post hoc analysis was based is the open-label design and lack of a comparison group for evaluation of change in bleeding over time. Given the documented variability and inconsistencies across studies in data collection methods and defining scheduled and unscheduled bleeding/spotting, meaningful comparisons among studies cannot be made. ${ }^{36}$ The current study approach to counting any scheduled bleeding days 
that continued into the next active pill pack as unscheduled bleeding/spotting days provides a "worst case" picture for unscheduled bleeding. Further, no correction to unscheduled bleeding/spotting was made for reported missed pill episodes. The exploratory subgroup analyses involving prior COC use, smoking status, BMI, and age had small sample sizes within the subgroups, and the outcomes of these analyses should be considered preliminary.

Future studies examining this extended-regimen COC should evaluate user satisfaction over time and reasons underlying contraceptive satisfaction or dissatisfaction as they relate to method adherence and continuation, as this may identify additional objectives for contraceptive counseling. Although awareness and acceptance of extended-regimen COC have increased, extended-regimen COC continues to be underused, ${ }^{12}$ even at contraceptive specialty clinics. ${ }^{16}$ Future studies that target increased awareness of extended-regimen COC among women and their HCPs, as well as increased HCP understanding of the broad usefulness of such regimens for improving women's QOL, are needed.

\section{Conclusion}

Extended-regimen COC with only four scheduled bleeds per year meets an important need among women seeking COC who prefer fewer menstrual cycles. The incidence and duration of bleeding with $84 / 7$ LNG/EE extended-regimen COC, including 7 days of low-dose EE during the traditional HFI, decreased over time. By the third and fourth cycles, more than one in five women reported no scheduled bleeding/spotting. By the second cycle, more than $80 \%$ of women reported no unscheduled bleeding or $\leq 6$ days of unscheduled bleeding during each 3-month cycle. Women considering this extended-regimen COC should be informed that those who complete at least one 91-day COC cycle will likely experience less bleeding in future cycles.

\section{Acknowledgments}

The authors wish to thank Lynanne McGuire, PhD, of MedVal Scientific Information Services, LLC, for providing medical writing and editorial assistance. This manuscript was prepared according to the International Society for Medical Publication Professionals' "Good Publication Practice for Communicating Company-Sponsored Medical Research: the GPP3 Guidelines". Medical writing assistance was provided by MedVal Scientific Information Services, LLC (Princeton, NJ, USA), and was funded by Teva Branded Pharmaceutical Products R\&D, Inc. (Frazer, PA, USA). This study was supported by Teva Pharmaceuticals, and Teva provided a full review of the article.

\section{Disclosure}

REN had a financial relationship (lecturer, member of advisory boards, and/or consultant) with Bayer-Schering Pharma, Endoceutics, Gedeon Richter, HRA Pharma, Merck Sharpe \& Dohme (MSD), Novo Nordisk, Pfizer Inc, Shionogi Limited, Teva/Theramex, and Zambon SpA. PLA had financial relationship (member of advisory boards, lecturer, and/or consultant) with Bayer, Effik, MSD, Smith \& Nephew, and Teva. JH is an employee of Teva Branded Pharmaceutical Products R\&D, Inc. MCM is a consultant for Teva Europe Women's Health Medical Affairs. The authors report no other conflicts of interest in this work.

\section{References}

1. Hooper DJ. Attitudes, awareness, compliance and preferences among hormonal contraception users: a global, cross-sectional, self-administered, online survey. Clin Drug Invest. 2010;30(11):749-763.

2. Egarter C, Frey Tirri B, Bitzer J, et al. Women's perceptions and reasons for choosing the pill, patch, or ring in the CHOICE study: a crosssectional survey of contraceptive method selection after counseling. BMC Women's Health. 2013;13:9.

3. Seval DL, Buckley T, Kuehl TJ, Sulak PJ. Attitudes and prescribing patterns of extended-cycle oral contraceptives. Contraception. 2011; 84(1):71-75.

4. Panicker S, Mann S, Shawe J, Stephenson J. Evolution of extended use of the combined oral contraceptive pill. J Fam Plann Reprod Health Care. 2014;40(2):133-141.

5. Edelman A, Micks E, Gallo MF, Jensen JT, Grimes DA. Continuous or extended cycle vs. cyclic use of combined hormonal contraceptives for contraception. Cochrane Database Syst Rev. 2014;(7):CD004695.

6. Mendoza N, Lobo P, Lertxundi R, et al. Extended regimens of combined hormonal contraception to reduce symptoms related to withdrawal bleeding and the hormone-free interval: a systematic review of randomised and observational studies. Eur J Contracept Reprod Health Care. 2014;19(5): 321-339.

7. Szarewski A, von Stenglin A, Rybowski S. Women's attitudes towards monthly bleeding: results of a global population-based survey. Eur $J$ Contracept Reprod Health Care. 2012;17(4):270-283.

8. Nappi RE, Fiala C, Chabbert-Buffet N, et al. Women's preferences for menstrual bleeding frequency: results of the Inconvenience Due to Women's Monthly Bleeding (ISY) survey. Eur J Contracept Reprod Health Care. 2016;21(3):242-250.

9. Fruzzetti F, Paoletti AM, Lombardo M, Carmignani A, Genazzani AR. Attitudes of Italian women concerning suppression of menstruation with oral contraceptives. Eur J Contracept Reprod Health Care. 2008; 13(2):153-157.

10. Ferrero S, Abbamonte LH, Giordano M, et al. What is the desired menstrual frequency of women without menstruation-related symptoms? Contraception. 2006;73(5):537-541.

11. Edelman A, Lew R, Cwiak C, Nichols M, Jensen J. Acceptability of contraceptive-induced amenorrhea in a racially diverse group of US women. Contraception. 2007;75(6):450-453.

12. Nappi RE, Kaunitz AM, Bitzer J. Extended regimen combined oral contraception: A review of evolving concepts and acceptance by women and clinicians. Eur J Contracept Reprod Health Care. 2016;21(2): 106-115. 
13. Frederick CE, Edelman A, Carlson NE, Rosenberg KD, Jensen JT. Extended-use oral contraceptives and medically induced amenorrhea: attitudes, knowledge and prescribing habits of physicians. Contraception. 2011;84(4):384-389.

14. Pompei LM, Fernandes CE, Steiner ML, Strufaldi R, Melo NR. Attitudes, knowledge and prescribing habits of Brazilian gynecologists regarding extended-cycle oral contraceptives. Gynecol Endocrinol. 2013;29(12):1071-1074

15. Wiegratz I, Galilaer K, Sanger N, Rody A, Kuhl H, Schleussner E. Prescribing preferences and personal experience of female gynaecologists in Germany and Austria regarding use of extended-cycle oral contraceptives. Eur J Contracept Reprod Health Care. 2010;15(6):405-412.

16. Sauer U, Mann S, Brima N, Stephenson J. Offering extended use of the combined contraceptive pill: a survey of specialist family planning services. Int J Womens Health. 2013;5:613-617.

17. Seasonique (levonorgestrel/ethinyl estradiol tablets and ethinyl estradiol tablets) [prescribing information]. Horsham, PA: Teva Branded Pharmaceutical Products R\&D Inc.; 2012.

18. London A, Jensen JT. Rationale for eliminating the hormone-free interval in modern oral contraceptives. Int J Gynaecol Obstet. 2016; 134(1):8-12.

19. Vandever MA, Kuehl TJ, Sulak PJ, et al. Evaluation of pituitary-ovarian axis suppression with three oral contraceptive regimens. Contraception. 2008;77(3):162-170.

20. Endrikat J, Gerlinger C, Plettig K, et al. A meta-analysis on the correlation between ovarian activity and the incidence of intermenstrual bleeding during low-dose oral contraceptive use. Gynecol Endocrinol. 2003;17(2):107-114.

21. Kaunitz AM, Portman DJ, Hait H, Reape KZ. Adding low-dose estrogen to the hormone-free interval: impact on bleeding patterns in users of a 91-day extended regimen oral contraceptive. Contraception. 2009; 79(5):350-355.

22. Anderson FD, Gibbons W, Portman D. Safety and efficacy of an extended-regimen oral contraceptive utilizing continuous low-dose ethinyl estradiol. Contraception. 2006;73(3):229-234.

23. Bachmann G, Korner P. Bleeding patterns associated with oral contraceptive use: a review of the literature. Contraception. 2007;76(3): 182-189.

24. Hickey M, Agarwal S. Unscheduled bleeding in combined oral contraceptive users: focus on extended-cycle and continuous-use regimens. J Fam Plann Reprod Health Care. 2009;35(4):245-248.
25. Howard B, Trussell J, Grubb E, Lage MJ. Comparison of pregnancy rates in users of extended and cyclic combined oral contraceptive (COC) regimens in the United States: a brief report. Contraception. 2014; 89(1):25-27.

26. Kroll R, Seidman L, Ricciotti N, Howard B, Weiss H. A phase 1, multicentre, open-label study to evaluate ovarian follicular activity and hormone levels with an extended-regimen combined oral contraceptive with low-dose ethinyl estradiol supplementation. Eur J Contracept Reprod Health Care. 2015;20(4):249-258.

27. DiLiberti CE, O'Leary CM, Hendy CH, Waters DH, Margolis MB. Steady-state pharmacokinetics of an extended-regimen oral contraceptive with continuous estrogen. Contraception. 2011;83(1):55-61.

28. Anderson FD, Feldman R, Reape KZ. Endometrial effects of a 91-day extended-regimen oral contraceptive with low-dose estrogen in place of placebo. Contraception. 2008;77(2):91-96.

29. Nappi RE, Paoletti AM, Volpe A, et al. Multinational, multicentre, randomised, open-label study evaluating the impact of a 91-day extended regimen combined oral contraceptive, compared with two 28-day traditional combined oral contraceptives, on haemostatic parameters in healthy women. Eur J Contracept Reprod Health Care. 2014;19(4):285-294.

30. Davis MG, Reape KZ, Hait H. A look at the long-term safety of an extended-regimen OC. J Fam Pract. 2010;59(5):E3.

31. Dehlendorf C, Krajewski C, Borrero S. Contraceptive counseling: best practices to ensure quality communication and enable effective contraceptive use. Clin Obstet Gynecol. 2014;57(4):659-673.

32. Sanchez-Borrego R, Garcia-Calvo C. Spanish women's attitudes towards menstruation and use of a continuous, daily use hormonal combined contraceptive regimen. Contraception. 2008;77(2):114-117.

33. Coffee AL, Sulak PJ, Kuehl TJ. Long-term assessment of symptomatology and satisfaction of an extended oral contraceptive regimen. Contraception. 2007;75(6):444-449.

34. Nelson AL. Communicating with patients about extended-cycle and continuous use of oral contraceptives. J Womens Health (Larchmt). 2007;16(4):463-470.

35. Dehlendorf C, Levy K, Kelley A, Grumbach K, Steinauer J. Women's preferences for contraceptive counseling and decision making. Contraception. 2013;88(2):250-256.

36. Mishell DR Jr, Guillebaud J, Westhoff C, et al. Recommendations for standardization of data collection and analysis of bleeding in combined hormone contraceptive trials. Contraception. 2007;75(1):11-15.
International Journal of Women's Health

\section{Publish your work in this journal}

The International Journal of Women's Health is an international, peerreviewed open-access journal publishing original research, reports, editorials, reviews and commentaries on all aspects of women's healthcare including gynecology, obstetrics, and breast cancer. The manuscript management system is completely online and includes

\section{Dovepress}

a very quick and fair peer-review system, which is all easy to use. Visit http://www.dovepress.com/testimonials.php to read real quotes from published authors. 